\title{
DRUG RESISTANT TACHYCARDIA AND FEVER IN A NEONATE: A CASE OF SYSTEMIC CANDIDIASIS
}

\section{Gunethilake K.M.T.B. ${ }^{1}$, Parthipan S. ${ }^{1}$, Vidanapathirana M. ${ }^{2}$, Beneragama D.H. ${ }^{3}$}

\author{
${ }^{1}$ Teaching Hospital, Baticaloa, Sri Lanka \\ ${ }^{2}$ Department of Forensic Medicine, ${ }^{3}$ Department of Pathology, Faculty of Medical Sciences, \\ University of Sri Jayewardenepura, Sri Lanka
}

\begin{abstract}
\section{Introduction}

Invasive fungal infection is a known cause of morbidity and mortality in the neonates especially in immunocompromised children. Demonstration of organisms by culture remains the gold standard for mycological diagnosis.
\end{abstract}

\section{Case report}

A neonate delivered at 34 weeks of gestation with a birth weight of $1800 \mathrm{~g}$ was admitted to a premature baby unit with breathing difficulty on day 05 after delivery. On examination, the child was febrile and had tachyarrhythmia. Blood culture showed a mixed growth of organisms and the echocardiogram was suggestive of a cardiac tumor. The condition of the neonate deteriorated in spite of treatment with antibiotics and supportive therapy and the child died 20 days after admission. At autopsy, a large vegetation was found on the tricuspid valve.

\section{Conclusion}

Fungal endocarditis should have been considered as a possible cause especially in the presence of large vegetations in the right side of the heart in neonates. The presence of fungal abscesses in the kidneys supported the systemic nature of the infection. Tachyarrhythmias may have been due to the involvement of the conduction system of the heart as vegetations were found in the area of AV node.
Key words: Candida albicans, systemic candidiasis, fungal endocarditis

Corresponding author:

mudithavidana@sjp.ac.lk

\section{INTRODUCTION}

Invasive fungal infection is a known cause of neonatal morbidity and mortality especially in the immunocompromised ${ }^{1}$. Early diagnosis and rapid initiation of effective treatment is a prerequisite for successful management. Culture remains the gold standard for mycological diagnosis ${ }^{2,3}$. However, as fungal cultures are not done routinely, the diagnosis can be delayed which can result in grave consequences.

Predisposing conditions for systemic fungal infection include prematurity, patients in the intensive care units, patients with implants, severe burns, children with congenital or acquired immunodeficiency, neutropenic patients, following hematopoietic stem cell transplantation or solid organ transplantation, and patients on immunosuppressive treatment. Candida, aspergillus and cryptococcus are among the common fungi that cause systemic fungal infection. 


\section{CASE REPORT}

A neonate delivered at 34 weeks of gestation with a birth weight of $1800 \mathrm{~g}$, was admitted with breathing difficulty on day 05 after delivery. The child developed fever spikes and tachyarrhythmia around the $8^{\text {th }}$ day following admission which was unresponsive to the standard drugs. Blood culture showed mixed growth of bacteria and the echocardiogram suggested a cardiac tumor.

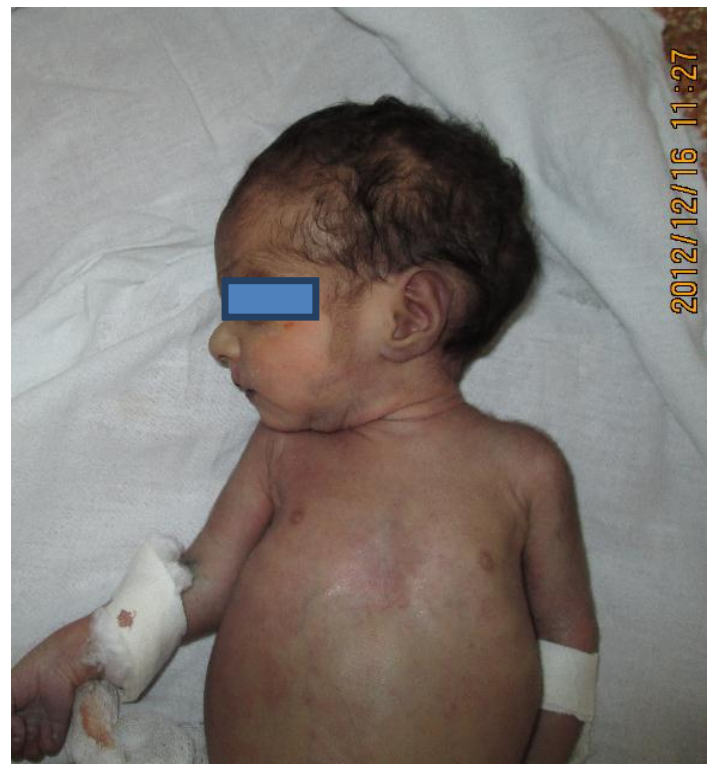

Figure 1: Cannula and distended abdomen
The child died on the 20 the day following admission. At autopsy, there was a cannula in-situ with a grossly distended abdomen (Figure 1) and a large, friable vegetation on the tricuspid valve (Figure 2).

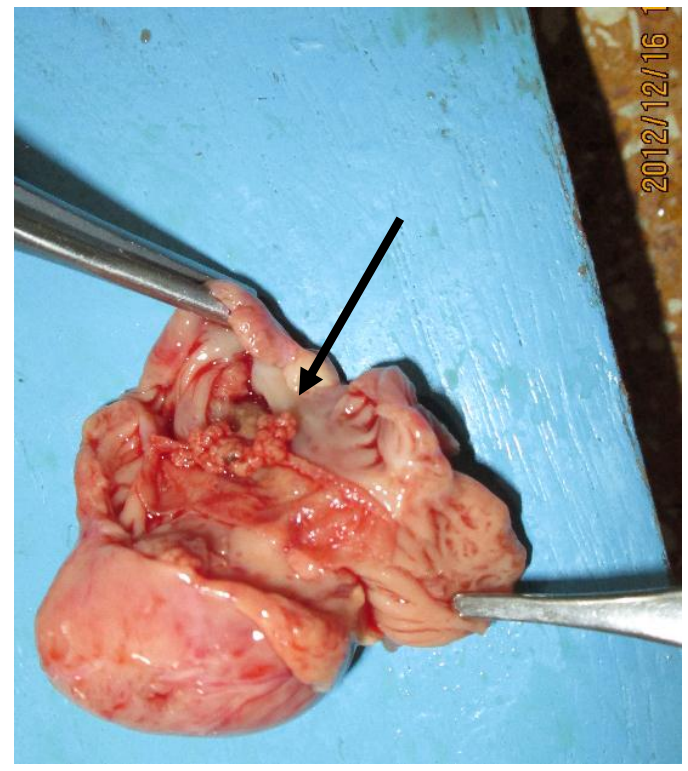

Figure 2: A large, friable vegetation on the tricuspid valve (arrow)

Histopathology of the vegetation showed hyphae and yeast forms (Figures 3, 4). Histopathology of the kidneys showed fungal micro-abscesses (Figure 5).

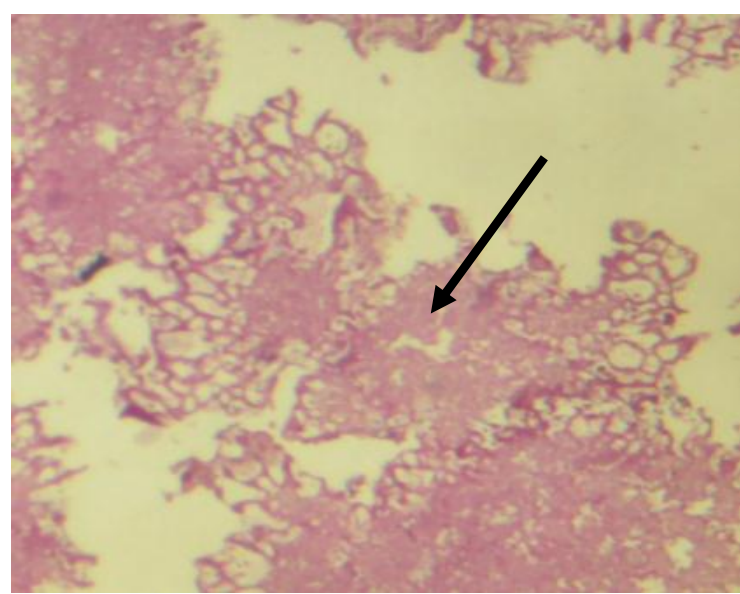

Figure 3: Candida organisms in the vegetation $(\mathrm{H} \& \mathrm{E} X \mathrm{X} 100)$

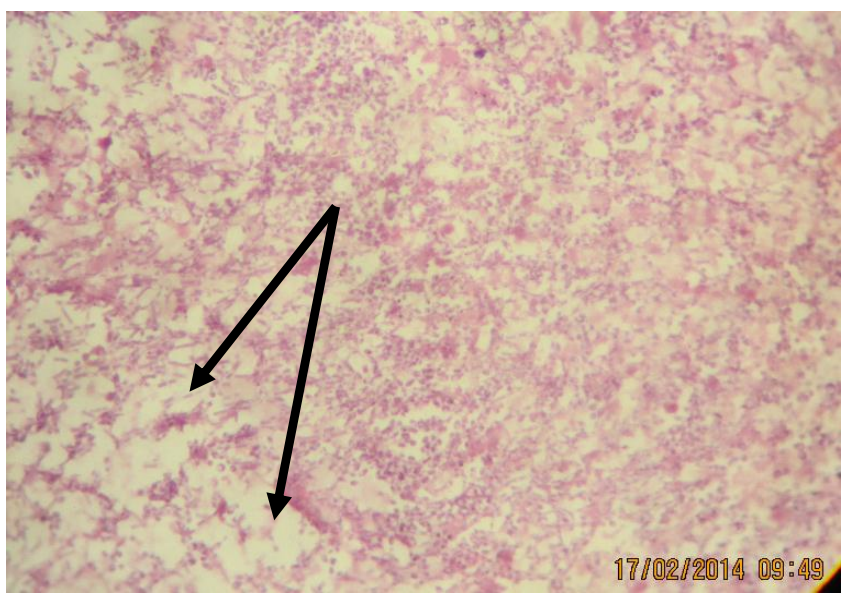

Figure 4: Candida hyphae and yeast forms in the vegetation (arrow) (H\&E X 100) 


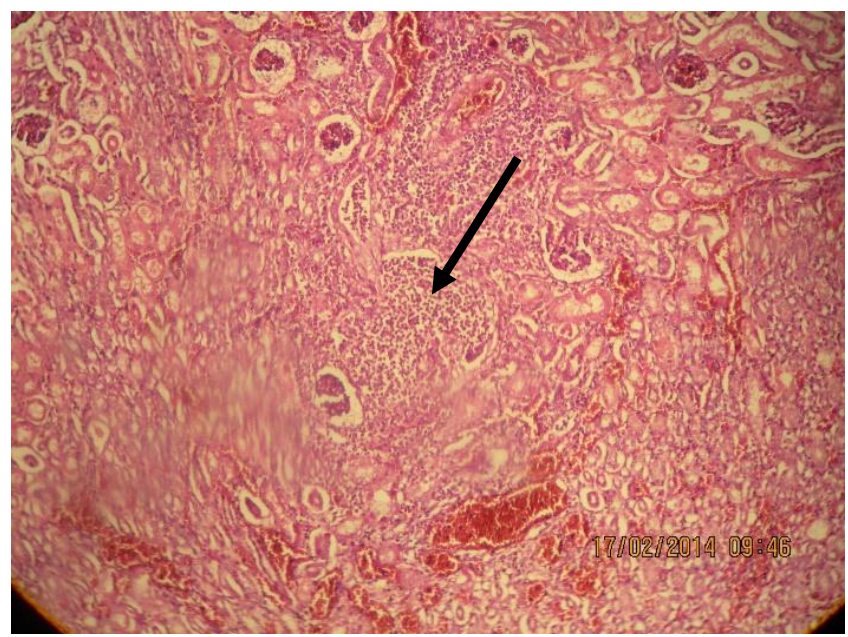

Figure 5: Candida micro abscess in kidney (H\&E X 100)

\section{DISCUSSION}

Candidiasis is defined as an infection or disease caused by a fungus of the genus Candida ${ }^{4}$. Systemic candidiasis is the most frequently encountered severe invasive opportunistic fungal infection. Major organs involved include the lungs, spleen, kidneys, liver, heart and the brain ${ }^{5}$. In the last few decades, the incidence of systemic candidiasis has increased resulting in an increased mortality rate ${ }^{4}$. Most of the cases are fatal due to the delay in diagnosis and initiation of effective therapy and are diagnosed only at postmortem examination ${ }^{6}$.

A diagnosis of invasive fungal infection requires a high index of suspicion ${ }^{7}$. The risk factors in this case included prematurity, low birth weight and insertion of an IV cannula. High fever for more than two weeks which was not responding to broad spectrum antibiotics was also supportive of a nonbacterial infection. Prematurity has been associated with a significant mortality and morbidity in patients with systemic candidiasis ${ }^{8}$.

False negative blood culture may be due to difficulty in interpretation by contamination with body commensals ${ }^{4}$. Special culture media are necessary for the best recovery of fungi ${ }^{7}$. Detection of candida antigen and antibodies by PCR and serology were found to be more sensitive in the diagnosis of systemic candidiasis than blood culture ${ }^{4}$.

At autopsy, ante-mortem specimens must be sent for histopathology and fungal culture for a definitive diagnosis ${ }^{7}$. If the specimens have not been sent for fungal culture, serology or PCR, histological examination supplemented by special histochemical stains such as PAS or methanamine silver stain can be helpful in the diagnosis of fungal infection.

The histology of sections from the large friable infective vegetation on the tricuspid valve confirmed a fungal vegetation with right sided fungal endocarditis. This large vegetation was misinterpreted as a cardiac tumour on echocardiogram examination which resulted in further delay in treatment. Tricuspid vegetations can emboli in to lungs. The lungs showed areas of hemorrhagic necrosis. Multiple fungal micro abscesses were seen in the kidneys containing slender filaments and yeast forms at the center surrounded by necrosis and polymorphs with fungal balls obstructing the pelvicalyceal system. Involvement of kidneys confirmed the systemic nature of the infection which may have been largely contributory to the fatal outcome in this case. ${ }^{6}$ Involvement of the conduction system of the heart by the vegetations which were found in the area of AV node may have resulted in the tachyarrhythmias.

In conclusion, a fungal aetiology must be considered in premature neonates with fever for over two weeks unresponsive to broad spectrum antibiotics. Early diagnosis by culture, serology or PCR and initiation of treatment will result in a significant reduction of mortality and morbidity especially in the presence of vegetations involving the tricuspid valve or the right side of the heart. 


\section{REFERENCES}

1. Jain A, Jain S, Rawat S. Emerging fungal infections among children: A review on its clinical manifestations, diagnosis, and prevention. J Pharm Bioallied Sci. 2010 OctDec; 2(4): 314-320.

2. Roilides E. Early diagnosis of invasive aspergillosis in infants and children. 2006, Vol. 44, No. 1: 199-205.

3. Hall KK, Lyman JA. Updated Review of Blood Culture Contamination. ClinMicrobiol Rev. 2006 October; 19(4): 788-802.

4. Gegic M, Numanovic F, Delibegovic Z, Tihic $\mathrm{N}$, Nurkic M, Hukic M. The importance of serological tests implementation in disseminated candidiasis diagnose. CollAntropol. 2013 Mar;37(1):157-63.

5. Hughes WT. Systemic candidiasis: a study of 109 fatal cases. Pediatr Infect Dis. 1982 JanFeb;1(1):11-8.
6. Mathai AM, Menezes RG, Naik R, Kanchan T, Kumar S, Bhat G, Chauhan A, Rai M. An autopsy case of renal candidiasis. J Forensic Leg Med. 2009 Jan;16(1):31-4.

7. Larbcharoensub N, Srisuma S, Ngernprasertsri T, Aroonroch R, Chongtrakool P, Santanirand P, Chirachariyavej T, Sirikulchayanonta V. Invasive fungal infection in Ramathibodi Hospital: a ten-year autopsy review. J Med Assoc Thai. 2007 Dec;90(12):2630-7.

8. Johnson DE, Thompson TR, Green TP, Ferrieri P. Systemic candidiasis in very lowbirth-weight infants (less than 1,500 grams). Pediatrics. 1984 Feb;73(2):138-43. 